\title{
Influência de dureza e pH na capacidade adsortiva de diuron em carvão ativado
}

\author{
Influence of hardness and $\mathrm{pH}$ on adsorption capacity of \\ diuron on activated carbon
}

Data de entrada: 08/06/2015

Data de aprovação: $22 / 09 / 2016$

Vanessa da Costa Gomes*/Bruno Moreira da Silva/Cristina Filomêna Pereira Rosa Paschoalato/Reinaldo Pisani Júnior DOI:10.4322/dae.2017.003

\section{Resumo}

A capacidade adsortiva do diuron em CAG de babaçu foi determinada experimentalmente em função do

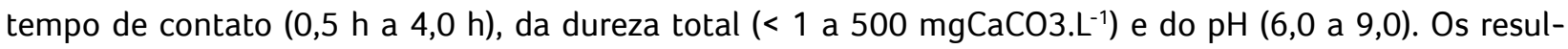
tados obtidos foram representados na forma de isotermas de adsorção segundo os modelos de Henry, Freundlich e Langmuir; dentre estes, a isoterma de Henry forneceu melhores ajustes. A constante de Henry tendeu a crescer em relação à dureza para pH de 6,0, enquanto que decresceu para os pH de 7,0 e 9,0, ou seja, a mudança de meio ácido para neutro ou básico fez com que as influências da dureza total em Cs e K se invertessem. Foi proposta uma correlação parametrizada na relação entre dureza total e pH para descrever esse comportamento e prever a constante de Henry com suficiente exatidão e, consequentemente, a capacidade adsortiva para diferentes condições, observados os limites de validade. Palavras-chave: Carvão Ativado Granular. Diuron. Dureza Total. Isotermas de Adsorção.

\section{Abstract}

The adsorptive capacity of diuron on granular activated carbon (GAC) was experimentally determined as a function of contact time ( $0.5 \mathrm{~h}$ to $4.0 \mathrm{~h}$ ), total hardness ( $<1$ to $500 \mathrm{mgCO}^{\left.-\mathrm{L}^{-1}\right)}$ and $\mathrm{pH}(6.0$ to 9.0). The results were represented as adsorption isotherms according to Henry, Freundlich and Langmuir models, among these, Henry isotherm provided the best data fit. The Henry's constant had tended to increase towards the hardness at pH 6.0, whereas it decreased to the $\mathrm{pH} 7.0$ and 9.0, i.e., the change in acid medium to neutral or alkaline caused that influences of the total hardness and $\mathrm{pH}$ in $\mathrm{Cs}$ and $\mathrm{K}$ were reversed. A parametrized in Hardness per $\mathrm{pH}$ ratio empirical correlation was proposed to describe the data behavior to forecast the Henry constant accurately, and consequently, to estimate adsorptive capacity in accordance with operational conditions and agreement of validity limits. Keywords: Activated Carbon Granular. Adsorption Isotherms. Diuron. Total Hardness.

\footnotetext{
Vanessa da Costa Gomes* - Bióloga e Pedagoga, Mestre em Tecnologia Ambiental pela Universidade de Ribeirão Preto, Doutoranda em Saúde Pública pela EERP/USP. E-mail: vanessaecobioayahoo.com.br.

Bruno Moreira da Silva - Engenheiro Químico, Mestrando em Tecnologia Ambiental da Universidade de Ribeirão Preto. E-mail: brunomoreira. engagmail.com.

Cristina Filomêna Pereira Rosa Paschoalato - Engenheira Química, Mestre e Doutora em Hidráulica e Saneamento pela EESC-USP, Docente do Curso de Engenharia Química e do Programa de Pós-Graduação em Tecnologia Ambiental da Universidade de Ribeirão Preto. E-mail: cpaschoaaunaerp.br.

*Endereço para correspondência: Rua Saldanha Marinho, 915 - Centro, Ribeirão Preto - SP CEP: 14010-060.
} 


\section{INTRODUÇÃO}

O crescimento da produção agrícola da cana-de-açúcar tem requerido a aplicação de diversos insumos químicos, sendo mais comum o uso dos herbicidas constituídos principalmente por diuron e hexazinona. Esses compostos são orgânicos clorados e nitrogenados e através da infiltração no solo e do escoamento superficial das águas pluviais podem contaminar as águas subterrâneas e superficiais.

Em função dessa vulnerabilidade, Paschoalato et al. (2009), Dantas et al. (2011) e Martinez et al. (2011) realizaram estudos para avaliar tecnologias de tratamento de água que promovam a remoção de diuron para atender ao padrão de potabilidade estabelecido pela vigente Portaria MS $n^{\circ}$ 2914 (BRASIL, 2011), com valor máximo permissível de $90 \mu \mathrm{g} \cdot \mathrm{L}^{-1}$ de diuron.

Existem algumas normas e padrões internacionais que limitam a concentração máxima desses herbicidas na água tratada. Segundo a norma canadense, a concentração máxima de diuron é de $150 \mu \mathrm{g} \cdot \mathrm{L}^{-1}$. Já a Comunidade Europeia recomenda a concentração máxima de agrotóxicos na água tratada de $0,5 \mu \mathrm{g} \cdot \mathrm{L}^{-1}$ e de qualquer agrotóxico de $0,1 \mu \mathrm{g} \cdot \mathrm{L}^{-1}$ (COUNCIL OF THE EUROPEAN UNION, 1998).

Segundo Dantas et al. (2011), as estações de tratamento de água destinada ao consumo humano que empregam a tecnologia convencional (ciclo completo) não estão preparadas para remover tais compostos orgânicos, considerados microcontaminantes. O estudo de Pádua (2009) destaca que o carvão ativado tem propriedades físicas e químicas que o categorizam como um adsorvente universal, que pode ser empregado nas ETA para remoção de diversos compostos orgânicos indesejavelmente presentes nas águas de mananciais, como carbamatos, carbofurano, diuron, hexazinona, glifosato, 2-4-D e cianotoxinas.

Martinez et al. (2011) determinaram a capacidade adsortiva de 13 carvões ativados de diferentes fabricantes, matérias-primas e características na remoção dos herbicidas diuron e hexazinona de águas sinteticamente contaminadas. Concluíram que o carvão ativado pulverizado (CAP) e o carvão ativado granular (CAG) de coco de babaçu foram os mais eficientes.

Já Paschoalato et al. (2009) avaliaram a remoção dos herbicidas diuron e hexazinona em água tratada utilizando a tecnologia ciclo completo sem e com adsorção em CAP e em CAG de babaçu. Comprovaram que o tratamento por ciclo completo não foi capaz de remover esses microcontaminantes. No entanto, quando complementada com adsorção com CAP ou CAG, a remoção foi quase completa, nas condições estudadas.

A escolha do adsorvente e a análise de viabilidade do uso da tecnologia de adsorção podem ser balizadas pela determinação experimental das isotermas de adsorção frente às condições operacionais e de qualidade da água afluente em ETA. Quanto maior a capacidade adsortiva, menor a quantidade de adsorvente necessária para uma aplicação específica, quer na forma de um leito móvel decorrente da elutriação das partículas de CAP, adicionadas na etapa de mistura rápida e tempo de contato estabelecido durante a coagulação e floculação, ou de um leito fixo de CAG, localizado após a filtração em areia.

A relação entre a quantidade de uma determinada substância (adsorvato) retida na superfície do sólido (adsorvente), ou seja, capacidade adsortiva, em função da concentração do soluto remanescente em solução no equilíbrio, é denominada isoterma de adsorção, cuja representação pode ser feita por modelos ou equações. O fenômeno da adsorção depende de diversos fatores, tais como potencial hidrogeniônico $(\mathrm{pH})$, dureza total, concentração do composto a ser adsorvido e das características do carvão (adsorvente) utilizado. A literatura apresenta diversos modelos para descrever isotermas de adsorção, nos quais os mais citados são os propostos por Freundlich e Langmuir (NETZ e ORTEGA, 2002). 
O estudo de influência do parâmetro pH na adsorção é importante na otimização dos sistemas de adsorção, particularmente para adsorção em fase líquida, uma vez que, nesses sistemas as cargas de superfície do adsorvente dependem do valor do pH (BABEL e KURNIAWAN, 2004).

Em função das diferentes características de solo, do escoamento superficial das águas de chuvas, as águas superficiais e subterrâneas podem apresentar diferentes concentrações de dureza e alcalinidade, podendo até apresentar variações sazonais. A dureza e a alcalinidade podem afetar a adsorção de micropoluentes sobre carvão, uma vez que o aumento da concentração de sal reduz as interações eletrostáticas tanto atrativas como repulsivas entre adsorvato-adsorvente ou devido a um efeito de blindagem. Além disso, a solubilidade de micropoluentes orgânicos depende da concentração de sal (PASTRANA-MARTÍNEZ, et al., 2010).

A adsorção é influenciada pela carga superficial do adsorvente e pelo grau de ionização do adsorvato, que por sua vez, são influenciados pelo $\mathrm{pH}$. $\mathrm{O}$ valor do $\mathrm{pH}$ da solução exerce influência sobre o processo global de adsorção e, particularmente, sobre a capacidade adsortiva, devido à influência nas propriedades de superfície do CAG. Um índice conveniente da tendência de uma superfície se tornar positiva ou negativamente carregada em função do $\mathrm{pH}$ é o valor de $\mathrm{pH}$ requerido para que a carga líquida do adsorvente seja nula, o chamado ponto de carga zero $\left(\mathrm{pH}_{\mathrm{zpc}}\right)$. Para valores de $\mathrm{pH}$ inferiores ao $\left(\mathrm{pH}_{\mathrm{zpc}}\right)$, a carga superficial é positiva e a adsorção de ânions é favorecida, e para valores de $\mathrm{pH}$ superiores ao $\left(\mathrm{pH}_{\mathrm{zpc}}\right)$ a carga superficial é negativa e a adsorção de cátions é favorecida (AL-DEGS et al, 2000).

Pastrana-Martínez et al. (2009) estudaram a adsorção de herbicida fluroxypyr (FLX) em diferentes tipos de carvão ativado, e os resultados mostraram que a retenção de FLX em carvões ativados decresceu com a elevação do $\mathrm{pH}$ de 2,0 para 10,0.
A dureza da água pode afetar a adsorção de contaminantes em carvões ativados; isso se deve ao fato de que a concentração de sais presentes no meio interfere na solubilidade de contaminantes orgânicos, dado que, quando a concentração dos sais aumenta, pode haver uma diminuição (fenômeno salting-out), ou aumento (fenômeno salting-in), na solubilidade do contaminante orgânico (BRESLOW e GUO, 1990; XIE, et al., 1997).

Os valores de $\mathrm{pH}$ e da dureza total na água potável distribuída pelas redes de abastecimento normalmente estão associados à incrustação de sais. A Portaria MS nº. 2914 (2011) estipula que a água potável deve ter $\mathrm{pH}$ no intervalo de $6,0 \mathrm{a}$ 9,5 e dureza total máxima de $500 \mathrm{mgCaCO}_{3} \cdot \mathrm{L}^{-1}$. Não há evidências claras de que a dureza total cause problemas sanitários. Contudo, em determinadas concentrações, pode ocasionar sabor, reduzir a formação de espumas e causar incrustações na rede e em trocadores de calor, devido principalmente à precipitação de sais de cálcio e magnésio, potencializada em temperaturas acima de $40^{\circ} \mathrm{C}$. Portanto, o limite estabelecido de 500 $\mathrm{mgCaCO}_{3} \cdot \mathrm{L}^{-1}$ está associado a questões econômicas, como aumento do consumo de sabões e interferência na qualidade de água para processos industriais (CORDEIRO, 2012).

Segundo Von Sperling (2005), embora não haja uma convenção formal, é usual empregar a seguinte classificação para representar a dureza da água: mole (0 - $\left.50 \mathrm{mg} \mathrm{CaCO}{ }_{3} \mathrm{LL}^{-1}\right)$; moderada (50 - $\left.150 \mathrm{mgCaCaCO}_{3} \mathrm{~L}^{-1}\right)$, dura $(150-300 \mathrm{mgCa}-$ $\left.\mathrm{CO}_{3} \cdot \mathrm{L}^{-1}\right)$ e muito dura ( $\left.>300 \mathrm{mgCaCO}_{3} \cdot \mathrm{L}^{-1}\right)$. Nesse sentido, para a investigação da influência do $\mathrm{pH}$ na capacidade adsortiva do diuron em CAG de babaçu, os pH utilizados foram de 6,0, 7,0 e 9,0 por estarem dentro da faixa estabelecida como qualidade aceitável de água potável. Da mesma forma, os valores de dureza total foram considerados de menor que 1, 100 e $500 \mathrm{mgCaCO}_{3} \cdot \mathrm{L}^{-1}$, para contemplar também as classificações referentes a águas mole, moderada e muito dura. 


\section{MATERIAL E MÉTODOS}

O método foi realizado em laboratório com ensaios em bancada e análises químicas diversas para caracterização e controle dos ensaios. 0 composto diuron foi quantificado pela técnica de Cromatografia a gás com detector de nitrogênio e fósforo (CG-DNP), em um equipamento Modelo 3800 CX, marca Varian, segundo método 507 (USEPA, 1995).

\subsection{Caracterização do carvão ativado granular}

O carvão ativado granular utilizado foi o carvão produzido a partir do coco de babaçu, por ser o mesmo utilizado em outros estudos realizados no Programa de Pesquisa em Saneamento Básico (PROSAB-5) (PADUA, 2009). Na caracterização do CAG foram determinados os seguintes parâmetros: número de iodo (ABNT- NBR 12073, 1991) índice de azul de metileno (JIS-K 1474, 1991), massa específica aparente (ABNT-NBR 12076, 1991), teor de umidade (ABNT-NBR 12077, 1991), teor de cinzas (ABNT-NBR 8112, 1986) e pH (ASTM, D3838, 1980).

\section{Determinação da Capacidade Adsortiva de Diuron em Carvão Ativado Granular}

O procedimento experimental para determinar as capacidades de saturação do carvão ativado em reter o diuron foi baseado na Norma ASTM D3860-98 (2003) JIS-K 1474 (1991). Essas normas estipulam o tempo de contado dinâmico entre as fases de $2 \mathrm{~h}$ para que o adsorvente seja saturado pelo adsorvato. No entanto, os tempos de contato utilizados no estudo foram de $0,5 \mathrm{~h}$ a 4,0 h, mantida a massa de CAG em aproximadamente $50 \mathrm{mg}$, com o intuito de verificar o tempo suficiente para estabelecer o equilíbrio. $A$ amostra de CAG foi processada em um moinho de alta energia com esferas de zircônia, para que $95 \%$ de sua massa fosse passante pela peneira com malha de 325 mesh $(0,044 \mathrm{~mm})$.
As massas de CAG utilizadas estiveram no intervalo de $0,0027 \mathrm{~g}$ a 0,0123 g, que foram alocadas em erlenmeyers de $250 \mathrm{~mL}$ para que os volumes totais das misturas fossem completadas até $200 \mathrm{~mL}$, pela adição de solução de diuron em água destilada com concentração inicial de 25 mg. $\mathrm{L}^{-1}, \mathrm{pH}(6,0,7,0$ ou 9,0) e dureza (inferior a 1, 100 e 500 mg CaCO3. $\mathrm{L}^{-1}$ ) previamente estabelecidos. $\mathrm{O} \mathrm{pH}$ dessas soluções foi mantido constante pelo emprego de soluções de tampão com fosfato (APHA, 2005), enquanto as durezas foram ajustadas e confirmadas por meio da determinação de dureza total por titrimetria, com EDTA 0,05M em presença do indicador de negro de Eriocromo T. Após a adição do CAG, a mistura foi realizada em mesa agitadora sob 100 rpm, de forma a promover o contato dinâmico com a solução a temperatura constante de $25^{\circ} \mathrm{C}$. Após decorrido o tempo de contato entre as fases líquida e sólida, as suspensões foram filtradas em membrana porosa com abertura média de 0,45 $\mu \mathrm{m}$ para que a concentração residual de diuron pudesse ser realizada pela técnica de cromatografia a gás com detetor de nitrogênio e fósforo (CG-DNP), segundo método 507 (USEPA, 1995).

Nos ensaios de adsorção em CAG foram utilizadas soluções tampão de fosfato de sódio para ajuste de pH em 6,0, 7,0 ou 9,0 e concentração inicial do adsorvato diuron de $25 \mathrm{mg}^{-L^{-1}}$ para diferentes massas de CAG, no intervalo de $2,7 \mathrm{mg}$ a $12,3 \mathrm{mg}$, mantidas em contato dinâmico mediante agitação a $25^{\circ} \mathrm{C}$ por 120 minutos. Em seguida, as soluções foram filtradas em membrana com abertura média de 0,45 $\mu \mathrm{m}$ para determinação das concentrações residuais de diuron. A medida do potencial hidrogeniônico foi feita em equipamento da marca Orion, modelo 230A, com eletrodo de vidro calomelano e calibrado com soluções, cujos $\mathrm{pH}$ eram de 4,0 e de 7,0. A dureza total foi determinada pelo emprego do método titrimétrico em presença de EDTA 0,05 $M$ e negro de Eriocromo T como indicador (APHA, 2005). As águas de estudo foram sinteticamente 
preparadas com adição de solução de carbonato de cálcio para obtenção de dureza total de 100 e 500 $\mathrm{mgCaCO}_{3} \cdot \mathrm{L}^{-1} \mathrm{em}$ água destilada.

\section{Modelos de Isotermas de Adsorção}

As equações utilizadas na tentativa de descrever o comportamento da capacidade adsortiva de diuron no CAG em função da concentração residual foram correspondentes aos modelos de Freundlich, Langmuir e Linear. O modelo de Freundlich considera a distribuição do adsorvato entre as fases sólida (adsorvente) e fluida (adsorvato) no equilíbrio (Equação 1), cuja linearização resulta na Equação 2 (NG et al., 2002; KURODA et al., 2005):

$$
C_{s}=K_{F} \cdot C_{e q}^{1 / n}
$$

Equação 1

$$
L n C_{s}=\operatorname{Ln} K_{F}+\frac{1}{n} \operatorname{Ln} C_{e q} \quad \text { Equação } 2
$$

em que Cs é a massa de adsorvato por unidade de massa de adsorvente $\left(\mathrm{mg}^{-1} \mathrm{~g}^{-1}\right), \mathrm{C}_{\text {eq }}$ é a concentração do adsorvato remanescente em solução, no equilíbrio (mg. $\left.\mathrm{L}^{-1}\right)$ e $\mathrm{K}_{\mathrm{F}}\left(m g^{1-1 / n} \cdot L^{1 / n} \cdot g^{-1}\right)$ e $1 / \mathrm{n}$ (adimensional) são constantes de ajuste. No modelo de Freundlich o coeficiente $\mathrm{K}_{\mathrm{F}}$ está relacionado à capacidade de adsorção do adsorvato pelo adsorvente, enquanto $n$ depende das características da adsorção. Para valores fixos de $C_{\text {eq }}$ e de $1 / n$, $\mathrm{C}_{\mathrm{s}}$ será tanto maior quanto maior for $\mathrm{KFe}$, para valores fixos de $\mathrm{K}_{\mathrm{F}}$ e de $\mathrm{C}_{\mathrm{eq}}$, energia de ligação entre adsorvente a adsorvato é crescente com o decréscimo do valor de $1 / \mathrm{n}$. Para valores de $1 / \mathrm{n}$ muito baixos, a capacidade de adsorção independe da concentração de adsorvato em solução e a isoterma de adsorção (relação entre $C_{s}$ e $C_{e q}$ ) se aproxima de uma reta horizontal, com $\mathrm{C}_{\mathrm{s}}$ aproximadamente constante, que é compatível com adsorção irreversível (quimissorção). Se o valor de $1 / n$ for muito elevado, a ligação na adsorção é fraca, com $\mathrm{C}_{\mathrm{s}}$ bastante dependente de $\mathrm{C}_{\mathrm{eq}}$. Quanto menor for o valor de $1 / n$, mais heterogênea deve ser a superfície adsorvente (ÖZCAN e ÖZCAN, 2004; WALKER e WEATHERLEY, 2001).

O modelo de Langmuir é termodinamicamente consistente, considera a superfície do adsorvente homogênea e assume energia de ligação uniforme, uma vez especificado o par adsorvato e adsorvente (CHOY et. al, 1999). A isoterma de Langmuir é representada na Equação 3, cuja linearização resulta na Equação 4:

$$
C_{s}=\frac{C_{0} \cdot C_{e q}}{K_{L}+C_{e q}}
$$

$$
\frac{1}{C_{s}}=\frac{K_{L}}{C_{0}} \cdot \frac{1}{C_{e q}}+\frac{1}{C_{0}}
$$

na qual $C_{s}$ é a massa de adsorvato por unidade de massa de adsorvente $\left(\mathrm{mg} \cdot \mathrm{g}^{-1}\right), \mathrm{C}_{\text {eq }}$ é a concentração do adsorvato remanescente em solução (mg. $\left.\mathrm{L}^{-1}\right), \mathrm{C}_{0}\left(\mathrm{mg} \cdot \mathrm{g}^{-1}\right)$ e $\mathrm{K}_{\mathrm{L}}\left(\mathrm{mg} \cdot \mathrm{L}^{-1}\right)$ são constantes a serem ajustadas.

O modelo linear, ou isoterma de Henry, é normalmente válido para sistemas diluídos, nos quais a curva de equilíbrio pode ser aproximada por uma reta que necessariamente passa pela origem dos eixos coordenados (Equação 5):

$$
C_{s}=K \cdot C_{e q} \quad \text { Equação } 5
$$

em que $\mathrm{C}_{\mathrm{s}}$ é a massa de adsorvato por unidade de massa de adsorvente (mg.g ${ }^{-1}$ ou $\left.\mu \mathrm{g} \cdot \mathrm{mg}^{-1}\right), \mathrm{K}$ é a constante do modelo ( $\left(\mathrm{L}^{-1}\right)$ e $\mathrm{C}_{\text {eq }}$ é a concentração do adsorvato no equilíbrio (mg. $\mathrm{L}^{-1}$ ou $\left.\mu \mathrm{g} \cdot \mathrm{L}^{-1}\right)$. A 
quantidade adsorvida remanescente foi calculada pela Equação 6:

$$
C_{s}=\frac{C_{0} \cdot V-C_{e q} \cdot V}{M}
$$

na qual $C_{s}$ é a quantidade adsorvida no sólido $\left(m g \cdot g^{-1}\right), C_{o}$ é a concentração inicial de diuron $\left(m g . L^{-1}\right), V$ é volume da amostra de água $(L)$ e $M$ é massa de CAG $\left(\mathrm{mg}^{-1} \mathrm{~g}^{-1}\right)$.

\section{Avaliação da Qualidade dos Ajustes}

As equações linearizadas (equações 2, 4 e 5) referentes aos modelos de Freundlich, Langmuir e Henry foram representadas graficamente para determinação dos coeficientes angulares e lineares. Posteriormente foi proposta uma correlação para prever as constantes a partir das condições experimentais estudadas de $\mathrm{pH}$ e dureza total.

A qualidade dos ajustes das capacidades de saturação experimentais $\left(C_{s}\right)$ às isotermas de Freundlich, Langmuir e Henry foi avaliada por meio da comparação dos valores dos coeficientes de determinação ( $R^{2}$ na Equação 7 ), visto que $R^{2}$ mais próximos a 1,0 associados a menores valores de RMSE indicam melhor qualidade de ajuste.

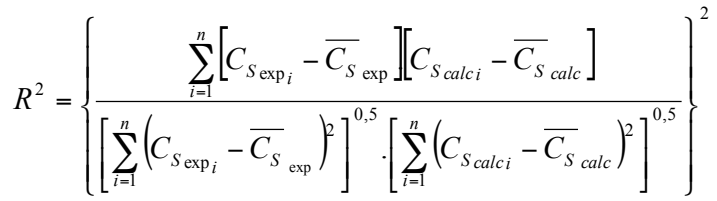

\section{Equação 7}

Nas quais $C_{S_{\exp }}$ é a capacidade adsortiva experimental, $C_{S_{c a l}}$ é a capacidade adsortiva cal- culada pelo modelo de isoterma, $\bar{C}_{S \text { exp }}$ é o valor médio das capacidades adsortivas experimentais e $\overline{C_{S}}$ calc é o valor médio das capacidades adsortivas calculadas.

Após a definição do melhor modelo de isoterma para representar $C_{s}$ em função de $C_{e q}$, foi proposta e testada uma função $K_{(p H, \text { Dureza })}$ para prever as constantes presentes no modelo de isoterma que melhor representou os resultados experimentais.

Os parâmetros de $K=f_{(p H, \text { Dureza })}$ foram determinados com a utilização do método dos mínimos quadrados, por intermédio da ferramenta Solver do programa Microsoft Excel. Novamente, a qualidade da representação foi avaliada dos valores de R2 e da raiz quadrada dos erros médios ao quadrado (RMSE) (equações 8 e 9 respectivamente), porém para as constantes existentes no modelo de isoterma escolhido. Já que é sabido que quanto menor for o valor de RMSE, melhor será o ajuste do modelo aos resultados a que se pretende representar.

A fim de verificar o grau de correlação linear entre as variáveis quantitativas independentes $\mathrm{pH} \mathrm{e}$ dureza total nos valores das constantes previstas para as isotermas, foram calculados os coeficientes de Pearson ( $r$ nas equações 10 e 11 respectivamente), se $r$ for igual a 1 , há uma correlação positiva perfeita entre as variáveis dependente e independente e se $r$ for igual a -1, verifica-se uma correlação negativa perfeita entre as variáveis, indicando que estas têm comportamentos opostos, ou seja, são inversamente relacionadas. Assim, foi possível priorizar as variáveis $\mathrm{pH}$ ou dureza para correlacionar os parâmetros presentes nas equações propostas. 
$R^{2}=\left\{\frac{\sum_{i=1}^{n}\left[K_{\exp _{i}}-\bar{K}_{\exp }\right]\left[K_{\text {calci }}-\bar{K}_{\text {calc }}\right]}{\left[\sum_{i=1}^{n}\left(K_{\exp _{i}}-\bar{K}_{\exp }\right)^{2}\right]^{0,5} \cdot\left[\sum_{i=1}^{n}\left(K_{\text {calci }}-\bar{K}_{\text {calc }}\right)^{2}\right]^{0,5}}\right\}^{2}$

Equação 8

$R M S E=\sqrt{\frac{\sum_{i=1}^{n}\left[K_{\exp _{i}}-K_{\text {calc }_{i}}\right]^{2}}{n}}$

Equação 9

$r=\frac{\sum\left(p H_{i}-\overline{p H}\right)\left(K_{\exp _{i}}-\bar{K}_{\exp }\right)}{\sqrt{\left(\sum\left(p H_{i}-\overline{p H}\right)^{2}\right)} \cdot \sqrt{\left(\sum\left(K_{\exp _{i}}-\bar{K}_{\exp }\right)^{2}\right)}}$

Equação 10

$r=\frac{\sum\left(\text { Dureza }_{i}-\overline{\text { Dureza }}\right)\left(K_{\exp _{i}}-\bar{K}_{\exp }\right)}{\sqrt{\left(\sum\left(\text { Dureza }_{i}-\overline{\text { Dureza }^{2}}\right) \cdot \sqrt{\left(\sum\left(K_{\exp _{i}}-\bar{K}_{\exp }\right)^{2}\right)}\right.}}$

Equação 11

Nas quais $K_{\exp _{i}}$ é a constante existente no modelo de isoterma obtida a partir dos resultados experimentais, $K_{\mathrm{cal}_{i}}$ é a constante do modelo de isoterma calculada pela correlação proposta ( $\left.K_{(p H, \text { Dureza })}\right), \bar{K}_{\text {exp }}$ é o valor médio das constantes existentes no modelo de isoterma obtidas experimentalmente e $\bar{K}_{\text {calc }}$ é o valor médio das constantes do modelo de isoterma calculadas pela correlação proposta e analogamente para as variáveis independentes $\mathrm{pH}$ e dureza.
Constatado o grau de correlação entre o pH e a dureza nas constantes presentes nas isoterma, foram testadas diferentes funções para representar a dependência em relação ao pH e dureza total.

\section{RESULTADOS E DISCUSSÕES}

A seguir são mostrados os resultados obtidos na caracterização do CAG, dos ensaios de adsorção e de ajuste dos modelos de Freundlich, Langmuir e Henry, além da correlação proposta para descrever as influências do $\mathrm{pH}$ e da dureza total nos parâmetros existentes nas equações e na constante capacidade adsortiva do CAG de babaçu.

\subsection{Resultados de Caracterização do Carvão Ativado Granular}

Na Tabela 1 são mostrados os parâmetros utilizados para caracterizar o CAG utilizado no estudo. Por exemplo, o número de iodo é um parâmetro utilizado para inferir sobre a quantidade de microporos existentes no CAG, visto que o tamanho da molécula de iodo é de aproximadamente 0,27 nm. É desejável que o índice de iodo seja superior a 500 $\mathrm{mg} \cdot \mathrm{g}^{-1}$ para a adsorção de solutos de massa molar da ordem de grandeza do iodo (253,8 g. mol-1) (JAGUARIBE et al., 2005; MARTINEZ et al., 2011). Já o índice de azul de metileno está associado à quantidade de mesoporo presente no CAG, porque sua molécula tem formato retangular com dimensões de $1,7 \mathrm{~nm}, 0,76 \mathrm{~nm}$ e 0,325 nm, com área projetada da molécula de aproximadamente 1,3 $\mathrm{nm} 2$ (HANG \& BRINDLEY, 1970). A molécula de azul de metileno tem secção transversal de cerca de 0,8 nm e estima-se que o diâmetro mínimo do poro que ela possa penetrar seja de $1,3 \mathrm{~nm}$.

A molécula de diuron tem massa molar de $233 \mathrm{~g}$. mol $^{-1}$, comprimento de 0,92 nm e largura de 0,49 $\mathrm{nm}$ (CHEN et al., 2004). Sendo assim, carvões com predominância de mesoporos em detrimento de microporos devem possuir maiores capacidades adsortivas para remover o diuron. 
Segundo Streat et al. (1995), os carvões ativados que possuem áreas superficiais superiores a 600 $\mathrm{m}^{2} \cdot \mathrm{g}^{-1}$ têm um alto potencial de adsorção. De acordo com Droste (1997), o valor da área superficial específica recomendado para os carvões ativados comerciais é de um limite mínimo de $500 \mathrm{~m}^{2} . \mathrm{g}^{-1}$. 0 CAG do presente estudo apresentou área superficial específica de $551,34 \mathrm{~m}^{2} \cdot \mathrm{g}^{-1}$ o que condiz com o limite mínimo recomendado pela referência.

Tabela 1: Caracterização do carvão CAG de babaçu selecionado para o estudo.

\begin{tabular}{|c|c|c|}
\hline Parâmetro & Unidade & CAG (Babaçu) \\
\hline Granulometria & $\begin{array}{c}2,38 \text { a } 1,41 \mathrm{~mm} \\
1,00 \text { a } 0,707 \mathrm{~mm} \\
0,177 \text { a } 0,420 \mathrm{~mm}\end{array}$ & $\begin{array}{l}(8 \times 14) \text { mesh } \\
(18 \times 25) \text { mesh } \\
(40 \times 80) \text { mesh }\end{array}$ \\
\hline Área superficial específica & $m^{2} \cdot g^{-1}$ & 551,34 \\
\hline $\mathrm{pH}$ & adimensional & $\begin{array}{c}\text { inicial } 6,40 \\
\text { final } 8,84 \pm 0,08\end{array}$ \\
\hline Teor de umidade & $\% \mathrm{~m} / \mathrm{m}$ & 0,33 \\
\hline Teor de cinzas & $\% \mathrm{~m} / \mathrm{m}$ & 9,90 \\
\hline Massa específica aparente & g.cm ${ }^{-3}$ & 0,49 \\
\hline Número de iodo & $\mathrm{mg} \cdot \mathrm{g}^{-1}$ & 136 \\
\hline Índice de azul de metileno & $\mathrm{mg} \cdot \mathrm{g}^{-1}$ & 260 \\
\hline
\end{tabular}

Nota-se que o CAG tem caráter alcalino, uma vez que $\mathrm{pH}$ da suspensão, formada por $10,00 \mathrm{~g}$ de CAG e $100 \mathrm{~mL}$ de água ultrapura, foi de 8,84 . Os resultados do teor de umidade, também se mostraram adequados de acordo com a recomendação da AWWA (2005), que determina que a umidade do carvão ativado não seja superior a $8 \%$. De acordo com Jaguaribe et al. (2005), o teor de cinzas de um carvão ativado comercial deve ser de até $15 \%$. Nos resultados obtidos, o teor de cinza foi de $9,90 \%$, abaixo do limite máximo recomendado. Os resultados da massa específica aparente se mostraram adequados de acordo com a recomendação da AWWA (2005), que estipula valores superiores a $0,25 \mathrm{~g} \cdot \mathrm{cm}^{-3}$.

Baçaoui et al. (2001) recomendaram que CAGs utilizados em sistemas de tratamento de água de- vam apresentar índice de azul de metileno igual ou superior a $180 \mathrm{mg} \cdot \mathrm{g}^{-1}$, condição obedecida, uma vez que valor obtido com o CAG de babaçu foi de $260 \mathrm{mg} \cdot \mathrm{g}^{-1}$. No entanto, o número de iodo obtido $\left(136,41 \mathrm{mg}^{-1}\right)$ foi inferior ao recomendado pela NBR 12073 (ABNT, 1991), que é de $600 \mathrm{mg}^{-1} \mathrm{~g}^{-1}$. Esse parâmetro, porém, está associado à presença de microporo, que não deve exercer influência significativa na adsorção do diuron, em função do tamanho da molécula. Em síntese, o carvão ativado granular de babaçu apresentou características potencialmente favoráveis para adsorção do Diuron, que se pretendeu avaliar por meio da determinação experimental das capacidades de adsortivas.

\subsection{Influência do Tempo de Contato Dinâmico entre Adsorvato e Adsorvente}

O tempo de contato dinâmico entre o adsorvato e o adsorvente utilizado no estudo e preconizado pela Norma ASTM D3860-98 (2003) foi de 2,0 h. A fim de comprovar se esse tempo foi suficiente para que o equilíbrio se estabelecesse, foram determinadas as capacidades adsortivas de diuron sobre CAG para tempos de contato no intervalo de 0,5 h a 4,0 h (Figuras 1 a 3), uma vez que, no equilíbrio, as CS deveriam ser essencialmente constantes para as diferentes condições abordadas.

Figura 1: Capacidade adosortiva de diuron sobre CAG de babaçu em função do tempo de contato e da dureza para o $\mathrm{pH}$ de 6,0.

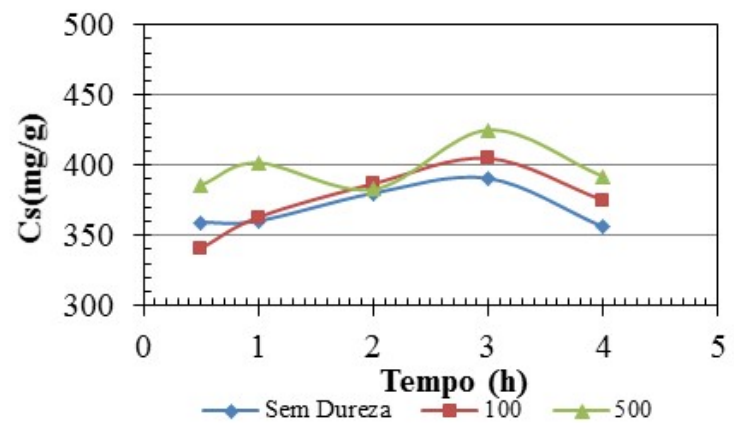


Figura 2: Capacidade adosortiva de diuron sobre CAG de babaçu em função do tempo de contato e da dureza para o $\mathrm{pH}$ de 7,0.

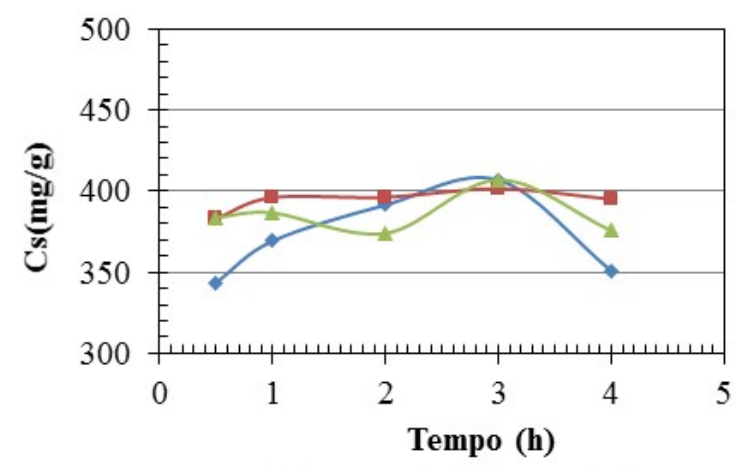

Figura 3: Capacidade adosortiva de diuron sobre CAG de babaçu em função do tempo de contato e da dureza para o $\mathrm{pH}$ de 9,0 .

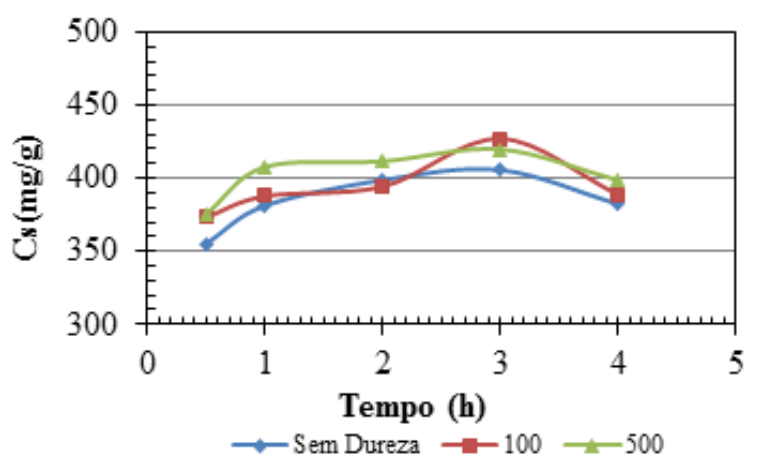

A norma JIS K 1474/1991 estipula que o tempo de contato entre o CAG e a solução seja de 2,0 h. Nota-se pelas Figuras 1 a 3 que a capacidade adsortiva do diuron em GAC foi praticamente constante para tempos superiores a 0,5 h. 0 desvio percentual máximo em relação ao valor médio, calculado pela média aritmética de Cs obtida 2,0, 3,0 e 4,0 h, foi de $6 \%$. Assim, o tempo de 2,0 h previsto na norma foi comprovadamente suficiente para que o equilíbrio entre o adsorvato e o adsorvente se estabelecesse. Além disso, pôde-se perceber que a capacidade adsortiva foi influenciada pelo $\mathrm{pH}$ e pela dureza total na condição estudada de massa de CAG constante.

\subsection{Ajuste dos Modelos de Isotermas de Freundlich, Langmuir e Henry}

Os resultados obtidos experimentalmente para CS e Ceq (Figuras 4, 5 e 6) foram ajustados aos modelos de Freundlich, Langmuir e Henry, com o intuito de descrever o comportamento da capacidade adsortiva do diuron em CAG de babaçu. $O$ critério de escolha do melhor modelo foi possuir maior coeficiente de determinação (R2) e fornecer resultados consistentes, como valores de CS e Ceq necessariamente positivos. Na Tabela 2 são mostrados os parâmetros ajustados para os três modelos de isoterma e do R2 nas condições estudadas. Nota-se pela tabela que os melhores ajustes foram obtidos com o modelo de Henry, uma vez que apresentaram os maiores valores de coeficientes de determinação R2 (no intervalo de 0,614 a 0,954) e não forneceram inconsistência como capacidades adsortivas ou concentrações de diuron no equilíbrio menores que zero.

Figura 4: Representação do modelo de Henry e dos valores experimentais da capacidade adsortiva do diuron em CAG de babaçu para pH de 6,0.

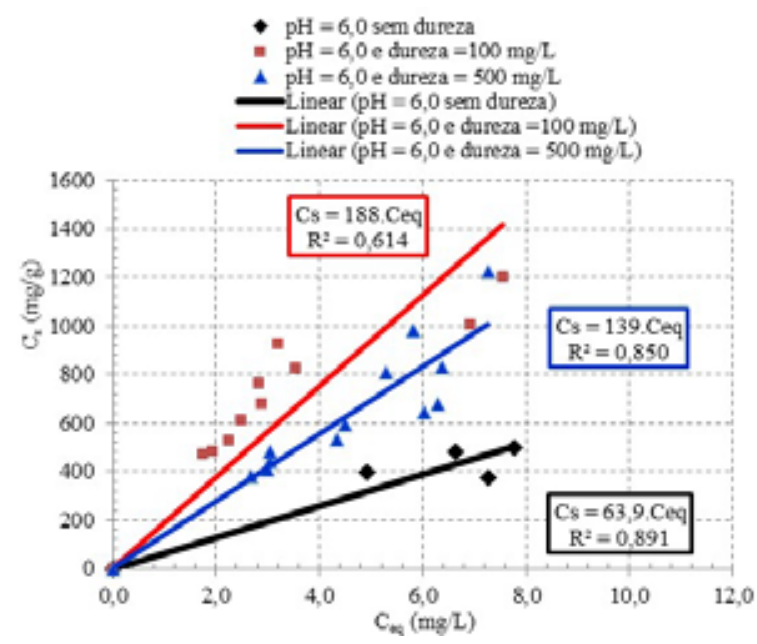


Figura 5: Representação do modelo de Henry e dos valores experimentais da capacidade adsortiva do diuron em CAG de babaçu para pH de 7,0.

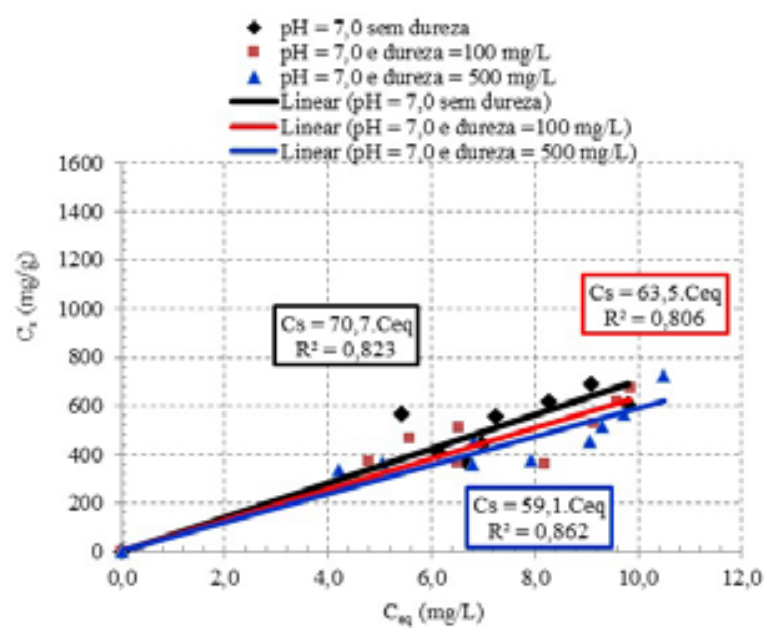

Figura 6: Representação do modelo de Henry e dos valores experimentais da capacidade adsortiva do diuron em CAG de babaçu para $\mathrm{pH}$ de 9,0.

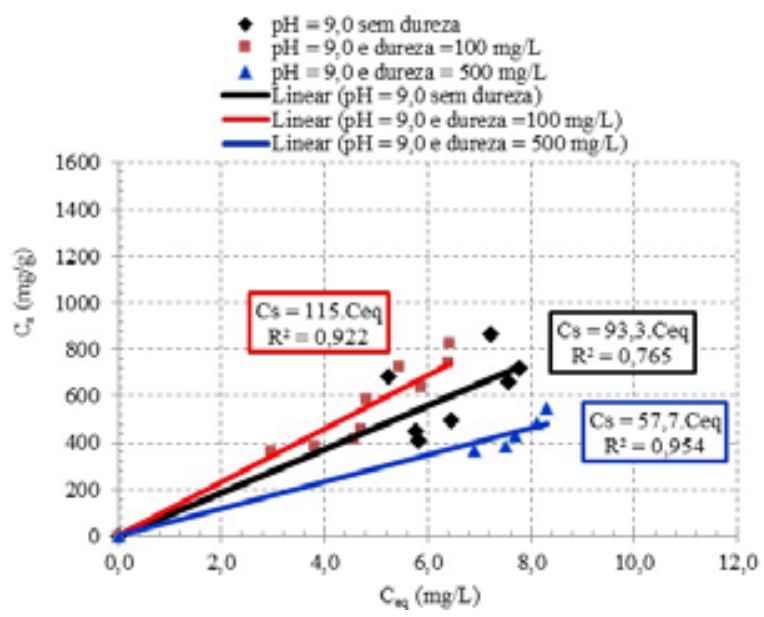

Percebe-se pela análise das Figuras 4 a 6 que tanto o $\mathrm{pH}$ como a dureza total interferiram nos valores da constante de Henry e consequentemente na capacidade adsortiva do diuron em CAG de babaçu, uma vez que a adsorção deve ser afetada pela carga superficial do adsorvente e pelo grau de ionização do adsorvato, que são influenciados pelo $\mathrm{pH}$ e pela quantidade de sal em solução representada pela dureza total. Isotermas obtidas para valores discretos de $\mathrm{pH}$ e dureza, porém, não permitem a interpolação dos valores para diferentes condições de dureza e pH daquelas obtidas experimentalmente. Sendo assim, é útil disponibilizar uma forma de obter as capacidades adsortivas $\left(\mathrm{C}_{\mathrm{s}}\right)$ para prever, ao menos de forma relativa, o impacto da mudança do $\mathrm{pH}$ e da dureza no desempenho de processos de adsorção envolvidos em tratamento de água ou efluentes. Por exemplo, a alteração da qualidade da água bruta com $\mathrm{pH}$ de 6,0 e dureza de $100 \mathrm{mgCaCO}_{3} \mathrm{~L}^{-1}$ para $\mathrm{pH}$ de 7,0 e dureza de $500 \mathrm{mgCaCO}_{3} \cdot \mathrm{L}^{-1}$ faz com que a constante de Henry passe de $188{\mathrm{~L} . \mathrm{g}^{-1}}^{\text {para }}$ 59,1 L.g ${ }^{-1}$, ou seja, mantida a concentração do diuron, a sua retenção será reduzida em aproximadamente $70 \%$.

Tabela 2: Resultados obtidos com a aplicação dos modelos de Freundlich, Langmuir e Henry.

\begin{tabular}{|c|c|c|c|c|c|c|c|c|c|}
\hline \multirow[t]{2}{*}{ pH } & \multirow{2}{*}{$\begin{array}{l}\text { Dureza total } \\
\left(\mathrm{mgCaCO}_{3} / \mathrm{L}\right)\end{array}$} & \multicolumn{3}{|c|}{ Freundlich } & \multicolumn{3}{|c|}{ Langmuir } & \multicolumn{2}{|c|}{ Henry } \\
\hline & & LnK $_{F}$ & $1 / n$ & $\mathbf{R}^{2}$ & $\mathrm{~K}_{\mathrm{L}} / \mathrm{C}_{0}$ & $1 / C_{0} \cdot 10^{3}$ & $\mathbf{R}^{2}$ & $\mathbf{K}_{\text {expi }}$ & $\mathbf{R}^{2}$ \\
\hline $\mathrm{pH} 6,0$ & $<1$ & 0,769 & 4,65 & 0,678 & 0,00997 & 0,685 & 0,595 & 63,9 & 0,894 \\
\hline $\mathrm{pH} 6,0$ & 100 & 0,610 & 5,87 & 0,861 & 0,00309 & 0,384 & 0,915 & 188 & 0,614 \\
\hline $\mathrm{pH} 6,0$ & 500 & 0,938 & 5,02 & 0,828 & 0,00648 & 0,185 & 0,881 & 139 & 0,850 \\
\hline $\mathrm{pH} 7,0$ & $<1$ & $-1,62$ & 13,1 & 0,390 & 0,00848 & 0,783 & 0,261 & 70,7 & 0,823 \\
\hline $\mathrm{pH} 7,0$ & 100 & 0,597 & 4,98 & 0,427 & 0,00797 & 1,04 & 0,355 & 63,5 & 0,806 \\
\hline $\mathrm{pH} 7,0$ & 500 & 0,662 & 4,78 & 0,667 & 0,00887 & 1,04 & 0,641 & 59,1 & 0,862 \\
\hline $\mathrm{pH} 9,0$ & $<1$ & 0,980 & 4,55 & 0,285 & 0,0103 & 0,138 & 0,253 & 93,3 & 0,765 \\
\hline $\mathrm{pH} 9,0$ & 100 & 1,12 & 4,54 & 0,832 & 0,00905 & $-0,006$ & 0,808 & 115 & 0,922 \\
\hline pH 9,0 & 500 & 2,16 & 1,67 & 0,880 & 0,0370 & $-2,52$ & 0,885 & 57,7 & 0,954 \\
\hline
\end{tabular}




\subsection{Proposição de Correlação e Avaliação da Qualidade do Ajuste}

De maneira geral, a constante de Henry tendeu a crescer em relação à dureza total para levemente ácido no pH de 6,0 (coeficiente de Pearson de 0,304), enquanto decresceu para os $\mathrm{pH}$ de $7,0 \mathrm{e}$ 9,0 (coeficiente de Pearson de $-0,890$ e - $-0,840$ respectivamente), ou seja a mudança de meio ácido para neutro ou básico fez com que a influência da dureza total em Cs e K se invertesse.

No entanto, para soluções de diuron sem adição de dureza total (inferior a $1 \mathrm{mg} . \mathrm{L}-1$ ), a interferência do $\mathrm{pH}$ acarretou no acréscimo de Ke favoreceu a interação adsorvato-adsorvente (coeficiente de Pearson de $+0,994)$. No entanto, a presença de dureza adicional fez com que essa tendência se invertesse. Isso ficou evidenciado nos resultados mostrados na Tabela 3, em que se puderam observar valores do coeficiente de Pearson positivos e negativos em função das condições operacionais.

Qualitativamente, os módulos de r obtidos no intervalo entre 0,30 e 0,994 (Tabela 3) são compatíveis com moderada a forte correlação entre as variáveis. Pois, para Cohen (1988), valores entre 0,10 e 0,29 indicam fraca correlação, entre 0,30 e 0,49, média, e entre 0,50 e 1 forte correlação. Dancey e Reidy (2006) apontaram para uma a seguinte categorização: $r$ de 0,10 a 0,30 (fraca), de 0,40 a 0,6 (moderada) e $r$ de 0,70 a 1,00 (forte).

Tabela 3: Resultados da constante de Henry obtidas a partir dos dados experimentais e coeficientes de correlação de Pearson em relação ao pH e à dureza total.

\begin{tabular}{|c|cc|c|}
\hline $\mathbf{p H}$ & $\begin{array}{c}\text { dureza } \\
\left.\mathbf{( m g ~ C a C O}_{\mathbf{3}} \mathbf{L} \mathbf{L}\right)\end{array}$ & $\begin{array}{c}\mathbf{K}_{\text {expj }} \\
\mathbf{( L / g )}\end{array}$ & $\begin{array}{c}\text { r em relação } \\
\text { à dureza }\end{array}$ \\
\hline 6,0 & 1 & 63,9 & \\
6,0 & 100 & 188 & $\mathbf{0 , 3 0 4}$ \\
6,0 & 500 & 139 & \\
\hline 7,0 & 1 & 70,7 & \\
7,0 & 100 & 63,5 & $-\mathbf{0 , 8 9 0}$ \\
7,0 & 500 & 59,1 & \\
\hline 9,0 & 1 & 93,3 & \\
9,0 & 100 & 115 & $-\mathbf{0 , 8 4 0}$ \\
\hline 9,0 & 500 & 57,7 & \\
\hline dureza & $\mathbf{p H}$ & $\mathbf{K}$ expj & $\mathbf{r}$ em relação à \\
(mg CaCO3/L) & $\mathbf{p H}$ & $\mathbf{( L / g )}$ & dureza \\
\hline 1 & 6,0 & 63,9 & \\
\hline 1 & 7,0 & 70,7 & $\mathbf{0 , 9 9 4}$ \\
\hline 1 & 9,0 & 93,3 & \\
\hline 100 & 6,0 & 188 & \\
\hline 100 & 7,0 & 63,5 & $\mathbf{- 0 , 4 1 9}$ \\
\hline 100 & 9,0 & 115 & \\
\hline 500 & 6,0 & 139 & \\
\hline 500 & 7,0 & 59,1 & $-\mathbf{0 , 7 6 6}$ \\
\hline 500 & 9,0 & 57,7 & \\
\hline
\end{tabular}

Diferentes funções foram testadas para representar a dependência da constante de Henry em função do pH e da dureza total. A mais simples e ao mesmo tempo mais satisfatória consistiu de uma equação quadrática explicitada na relação entre a dureza total e o pH (Equação 12), cujos parâmetros foram dependentes apenas do $\mathrm{pH}$ (equações 13 a 15).

$K_{\text {calc }(L / g)}=a_{(p H)} \cdot\left(\frac{\text { Dureza }}{p H}\right)^{2}+b_{(p H)} \cdot\left(\frac{\text { Dureza }}{p H}\right)+c_{(p H)}$

Equação 12

para o intervalo de $0,111 \mathrm{mgCaCO}{ }_{3} \cdot \mathrm{L}^{-1} \leq$ Dureza/ $\mathrm{pH} \leq 83,3 \mathrm{mgCaCO}_{3} \cdot \mathrm{L}^{-1}$, com a, b e c obtidos pelas equações 13, 14 e 15: 
$a_{(p H)}=-4,5927 \cdot 10^{-2} \cdot(p H)^{2}+7,0239 \cdot 10^{-1} \cdot(p H)-2,6602$

Equação 13

$b_{(p H)}=3,8012 \cdot(p H)^{2}-59,2046 \cdot(p H)+227,6$

Equação 14

$c_{(p H)}=0,8991 \cdot(p H)^{2}-3,2745 \cdot(p H)+49,656$

Equação 15

válidas para o intervalo de 6,0 $\leq \mathrm{pH} \leq 9,0 . \mathrm{Na}$ Tabela 4 são mostradas as constantes de Henry obtidas a partir dos resultados experimentais e as previstas pela correlação proposta (equações 12 a 15), assim como os parâmetros utilizados para avaliar a qualidade do ajuste.

Tabela 4: Constantes de Henry obtidas a partir dos resultados experimentais e calculadas pela correlação proposta (equações 13 a 16).

\begin{tabular}{|c|c|c|c|c|c|}
\hline $\mathrm{pH}$ & $\begin{array}{c}\text { Dureza } \\
(\mathrm{mg} \mathrm{CaCO} / \mathrm{L})\end{array}$ & $\begin{array}{l}K_{\text {expj }} \\
(\mathrm{L} / \mathrm{g})\end{array}$ & $\begin{array}{l}\mathrm{K}_{\text {calci }} \\
(\mathrm{L} / \mathrm{g})\end{array}$ & $\mathbf{R}^{2}$ & RMSE (L/g) \\
\hline $\begin{array}{l}6,0 \\
6,0 \\
6,0\end{array}$ & $\begin{array}{c}1 \\
100 \\
500\end{array}$ & $\begin{array}{l}63,9 \\
188 \\
139\end{array}$ & $\begin{array}{l}63,9 \\
188 \\
141\end{array}$ & 0,9997 & 1,31 \\
\hline $\begin{array}{l}7,0 \\
7,0 \\
7,0\end{array}$ & $\begin{array}{c}1 \\
100 \\
500\end{array}$ & $\begin{array}{l}70,7 \\
63,5 \\
59,1\end{array}$ & $\begin{array}{l}70,7 \\
63,8 \\
61,0\end{array}$ & 0,9909 & 1,11 \\
\hline $\begin{array}{l}9,0 \\
9,0 \\
9,0\end{array}$ & $\begin{array}{c}1 \\
100 \\
500\end{array}$ & $\begin{array}{c}93,3 \\
115 \\
57,7\end{array}$ & $\begin{array}{c}93,3 \\
115 \\
59,1\end{array}$ & 0,9998 & 0,845 \\
\hline
\end{tabular}

Nota-se pelos resultados da Tabela 4 que a correlação proposta foi plenamente satisfatória em representar a dependência da constante de Henry em relação ao $\mathrm{pH}$ e à dureza total, tanto em comportamento como em módulo, em razão dos coeficientes de determinação bastante próximos a 1 e de raízes quadradas dos erros médios ao quadrado desprezíveis em relação aos valores previstos para $\mathrm{K}$ e consequentemente a curva de equilíbrio decorrente.

Assim, é possível inferir sobre o impacto de alterações no $\mathrm{pH}$ e na dureza total sobre o processo de adsorção do diuron sobre o CAG dentro do intervalo de amplitude estabelecido pela Portaria MS 2914 (2011). Na Figura 7 são mostrados valores da constante de Henry estimados pela correlação proposta (equações 12 a 15), em que se pode observar a influência simultânea do $\mathrm{pH}$ e da dureza total.

Figura 7: Constante de Henry prevista pela correlação proposta em função do pH e da dureza total.

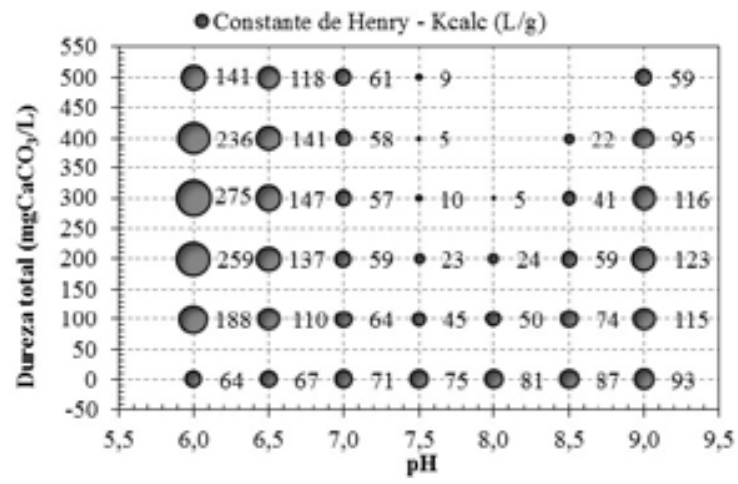


Por exemplo, em sistemas de tratamento de água por ciclo completo que utilizam como coagulante os sais de alumínio, o pH de coagulação normalmente é de 6,5 associado a uma dureza total típica de $50 \mathrm{mgCaCO}_{3} \cdot \mathrm{L}^{-1}$ resultando na capacidade adsortiva de 90,5 L.g ${ }^{-1}$. Na alteração de qualidade da água bruta que resulte na operação da ETA no $\mathrm{pH}$ de 7,4 e dureza total de $200 \mathrm{mgCaCO}_{3} \cdot \mathrm{L}^{-1}$, ainda compatível com a região de coagulação de sais de alumínio, a constante de Henry passaria a ser de 27 L.g ${ }^{-1}$, ou seja, uma redução de aproximadamente $70 \%$, que impactaria negativamente na remoção do diuron por meio de sistemas de adsorção em leitos fixos com CAG ou em leitos deslizantes com CAP.

\section{CONCLUSÕES}

O tempo de 2,0 h foi suficiente para que o equilíbrio entre o adsorvato e o adsorvente fosse estabelecido, uma vez que não houve acréscimo considerável na capacidade adsortiva para tempos de contato dinâmico superiores a esse valor.

Os melhores ajustes foram obtidos com o modelo de Henry, com os maiores valores de coeficientes de determinação $R^{2}$ (no intervalo de 0,614 a $0,954)$ e não forneceram inconsistência como capacidades adsortivas ou concentrações de diuron no equilíbrio menores que zero.

A correlação proposta por uma equação quadrática explicitada na relação entre dureza total e o pH foi plenamente satisfatória em representar a dependência da constante de Henry, tanto em comportamento como em módulo, com coeficientes de determinação próximos a 1 e de raízes quadradas dos erros médios ao quadrado desprezíveis em relação aos valores previstos para $\mathrm{K}$ e consequentemente a curva de equilíbrio decorrente.

$\mathrm{O} \mathrm{pH}$ e a dureza total interferiram acentuadamente na constante de Henry e consequentemente, na capacidade adsortiva do diuron em CAG de babaçu que, a depender das alterações na qualidade da água, pode impactar favorável ou desfavoravelmente na remoção do diuron em sistemas de adsorção em leitos fixos com CAG ou em leitos deslizantes com CAP.

\section{REFERÊNCIAS}

AL-DEGS, Y.; ALLEN, STEPHEN; KHRAISHEH, M.. Effect of Carbon Surface Chemistry on the Removal of Reactive Dyes from Textile Effluent. Water Research, v. 34, n³ 3. p. 927-935, 2000.

AMERICAN SOCIETY OF TESTING AND MATERIALS (ASTM). D 3838-1980: Standard Test Method for pH of Activated Carbon. Estados Unidos, 1999.

AMERICAN SOCIETY OF TESTING AND MATERIALS (ASTM) D386098: Standard practice for determination of adsorptive capacity of activated carbon by aqueous phase isotherm technique. Estados Unidos, 2003.

AMERICAN WATER WORKS ASSOCIATION (AWWA) UNITED STATES. Treatment Plant Design. Estados Unidos: Editora McGraw-Hill, 2005, 4a edição.

AMERICAN PUBLIC HEALTH ASSOCIATION (APHA); AMERICAN WATER WORK ASSOCIATION(AWWA); WATER ENVIRONMENT FEDERATION (WPCF). Standard methods for the examination of water and wastewater. Washington: American Public Health Association, 2005.21a ed,p. 1368.

ASSOCIAÇÃO BRASILEIRA DE NORMAS TÉCNICAS (ABNT), NBR 8112: Carvão Vegetal. Análise imediata. Código Secundário: MB 1857. Rio de Janeiro, 1986.

ASSOCIAÇÃO BRASILEIRA DE NORMAS TÉCNICAS (ABNT), NBR 12073: Carvão ativado pulverizado. Determinação do número de iodo. Código Secundário: MB 3410. Rio de Janeiro, 1991.

ASSOCIAÇÃO BRASILEIRA DE NORMAS TÉCNICAS (ABNT), NBR 12076: Carvão ativado pulverizado. Determinação da massa específica aparente. Código Secundário: MB 3413. Rio de Janeiro, 1991.

ASSOCIAÇÃO BRASILEIRA DE NORMAS TÉCNICAS (ABNT), NBR 12077: Carvão ativado pulverizado. Determinação da umidade. Código Secundário: MB 3414. Rio de Janeiro, 1991.

BABEL, S.; KURNIAWAN, T. A.. Removal from synthetic wastewater using coconut shell charcoal and commercial activated carbon modified with oxidizing agents and/or chitosan. Chemosphere, v. 54, p. 951-967, 2004.

BAÇAOUI, A.; YAACOUBI, A.; DAHBI, A.; BENNOUNA, C.; PHAN TAN LUU, R.; MALDONADO-HODAR, F.; RIVERAUTIRLLA, L; MORENO-CASTILLA, C.. Optimization of conditions for the preparation of activated carbons from olive-waste cakes. Carbons, v.39, n.3, p. 425-432, 2001. 
BRASIL. Ministério da Saúde. Portaria n² 2914, de 12 de dezembro de 2011. Publicado no Diário Oficial da União, Brasília, DF. 14 dez. 2011. Disponível em: <http://bvsms.saude.gov.br/bvs/ saudelegis/gm/2011/prt2914_12_12_2011.html> Acesso em 22 jul. 2014.

BRESLOW, R.; GUO, T.. Surface-tension measurements show that chaotropic salting-in denaturants are not just water-structure breakers. Proceedings of the National Academy of Sciences of the United States of America. v. 87, n 1, p.167-169, 1990.

COHEN, J. Statistical power analysis for the behavioral sciences. Hillsdale, NJ: Lawrence Earlbaum Associates, 1988, 2 ed.

CORDEIRO, M. M.; GADELHA, C. L. M.; BOMFIM, E. O.; SILVA, T. C.. Variações da dureza da água captada de poços do aquífero Beberibe na cidade de João Pessoa-PB. Revista Brasileira de Recursos Hídricos, v. 17, n 4, p. 239-247, Out/Dez 2012.

COUNCIL OF THE EUROPEAN UNION. Council Directive 98/83/EC Quality of Water intended for Human Consumption. Official Journal of the European Communities, 1998.

CHEN, S. S.; TAYLOR, J. S.; MULFORD, L. A.; NORRIS, C. D.. Influences of molecular weight, molecular size, flux, and recovery for aromatic pesticide removal by nanofiltration membranes. Desalinisation, v. 160, p. 103-111, 2004.

CHOY, K. K. H., MCKAY, G., PORTER, J. F.. Sorption of acid dyes from effluents using activated carbon. Resources Conservation and Recycling. v. 27, n.1-2, p. 57-71, 1999.

DANCEY, C; REIDY, J.. Estatística sem matemática para psicologia: usando SPSS para Windows. Porto Alegre: Artmed, 2006.

DANTAS, A. D. B; PASCHOALATO, C. F. P. R. P.; MARTINEZ, M. S.; BALLEJO, R. R.; DI BERNARDO, L.. Removal of diuron and hexazinone from guarany aquifer groundwater. Braz. J. Chem. Eng. v. 28, n. 3, p. 415-424, 2011.

DROSTE, R. L. Theory and practice of water and wastewater. Nova lorque: John Wiley \& Sons, 1997.

HANG, P. T. BRINDLEY, G. W.. Methylene blue absorption by clay minerals determination of surface areas and cation exchange capacities (clay-organic studies XVIII). Clays and Clay Minerals. v. 18, p. 203-212, 1970.

JAGUARIBE, E. F.; MEDEIROS, L. L.; BARRETO, M. C. S. and ARAUJO, L. P.. The performance of activated carbons from sugarcane bagasse, babassu, and coconut shells in removing residual chlorine. Braz. J. Chem. Eng., v. 22, n.1, p. 41-47, 2005.

JAPANESE INDUSTRIAL STANDARD (JIS) K 1474. Test methods for activated carbon. Japão, p. 47, 1991.

KURODA, E. K.; ALBUQUUERQUU JÚNIOR, E. C.; DI BERNARDO, L.; TROFINO, J. C.. Caracterização e escolha do tipo de carvão ativado a ser empregado no tratamento de águas contendo microcistinas. In: $23^{\circ}$ CONGRESSO BRASILEIRO DE ENGENHARIA SANITÁRIA E AMBIENTAL, 2005, Anais, Campo Grande-MS, ABES.
MARTINEZ, M. S., PIZA, A. V. T.; DANTAS, A. D. D.; PASCHOALATO, C. F. P. R.; DI BERNARDO, L.. Remoção dos herbicidas diuron e hexazinona de água por adsorção em carvão ativado. Revista DAE. v.185, p. 35-39, 2011.

NETZ, P. A.; ORTEGA, G. G. Fundamentos de Físico-Química: uma abordagem conceitual para as ciências farmacêuticas. Porto Alegre: Artmed, 2002.

NG, C.; LOSSO, J.N.; MARSHALL, W.E.; RAO, R.M.. Freundlich adsorption isotherms of agricultural by-productbased powdered activated carbons in a geosmin-water system. Bioresource Technology, v. 85, n. 2, p.131-135, 2002.

ÖZCAN, A. S; ÖZCAN, A. Adsorption of Acid Dyes from Aqueous Solutions Onto Acid-Activated Bentonite. Journal of Colloid and Interface Science, v. 276, p. 39-46, 2004.

PÁDUA, V. L. Programa de Pesquisa em Saneamento Básico (PROS$A B$ 5). Remoção de microrganismos emergentes e microcontaminantes orgânicos no tratamento de água para o consumo humano. Rio de Janeiro: ABES, 2009, p.221-232.

PASCHOALATO, C. F. P. R.; DANTAS, A. D. B.; ROSA, I. D. A.; FALEIROS, R. J. R.; DI BERNARDO, L.. Uso de carvão ativado para remoção dos herbicidas diuron e hexazinona de água. Revista DAE. v.179, p. 34-41, 2009.

PASTRANA-MARTÍNEZ, L.M.: LÓPEZ-RAMÓN, M.V.; MORENO-CASTILLA, C.. Adsorption and thermal desorption of the herbicide fluroxypyr on activated carbon fibers and cloth at different $\mathrm{pH}$ values. Journal of Colloid and Interface Science, 331. p. 2-7, 2009.

PASTRANA-MARTÍNEZ, L. M.; LÓPEZ-RAMÓN M.V.; FONTECHA-CÁMARA, M.A.; MORENO-CASTILLA, C.. Batch and column adsorption of herbicide fluroxypyr on different types of activated carbons from water with varied degrees of hardness and alkalinity. Water Research, v.44, p. 879-885, 2010.

STREAT, M.; PATRICK, W. J.; PEREZ CAMPORRO, M. J.. Sorption of phenol and para-chlorophenol from water using conventional and novel activated carbons. Water Research, v. 29, n. 2, p. 467472, 1995.

UNITED STATES ENVIRONMENTAL PROTECTION AGENCY. Method 507 Determination of Nitrogen and Phosphorus contaning pesticides in water by gas chromatography with a Nitrogen-Phosphorus Detector. J. W. Munch, 1995.

VON SPERLING, M.. Princípios do tratamento biológico de águas residuárias: Introdução à qualidade das águas e ao tratamento de esgotos. v.1. Belo Horizonte: Editora Universitária, 2005, $3^{\mathrm{a}} \mathrm{ed}$.

XIE, W. H.; SHIU, W-Y; MACKAY, D.. A review of the effect of salts on the solubility of organic compounds in seawater. Marine Environmental Research. v.44, n.4, p. 429-444, 1997.

WALKER, G. M.; WEATHERLEY, L. R. Adsorption of Dyes from Aqueous Solution - the Effect of Adsorbent Pore Size Distribuition and Dye Aggregation. Chemical Engineering Journal, v. 83, p. 201-206, 2001. 